\title{
Highly effective NK cells are associated with good prognosis in patients with metastatic prostate cancer
}

\author{
Christine Pasero ${ }^{1,3}$, Gwenaëlle Gravis ${ }^{3}$, Samuel Granjeaud ${ }^{1,3}$, Mathilde Guerin ${ }^{1,3}$, \\ Jeanne Thomassin-Piana ${ }^{3}$, Palma Rocchi ${ }^{1,3}$, Naji Salem ${ }^{3}$, Jochen Walz ${ }^{3}$, Alessandro \\ Moretta $^{4}$ and Daniel Olive ${ }^{1,2,3}$ \\ ${ }^{1}$ Centre de Recherche en Cancérologie de Marseille, Inserm, Marseille, France \\ ${ }^{2}$ Aix Marseille Université, Marseille, France \\ ${ }^{3}$ Institut Paoli-Calmettes, Marseille, France \\ 4 Dipartimento di Medicina Sperimentale (D.I.ME.S.), Università di Genova, Genova, Italy \\ Correspondence to: Daniel Olive, email: daniel.olive@inserm.fr \\ Christine Pasero, email: christine.pasero@inserm.fr
}

Keywords: natural killer cells, prostate cancer, metastases, survival, castration resistance

Received: December 21,2014 Accepted: April 10,2015 Published: April 29, 2015

This is an open-access article distributed under the terms of the Creative Commons Attribution License, which permits unrestricted use, distribution, and reproduction in any medium, provided the original author and source are credited.

ABSTRACT

Clinical outcome of patients with metastatic prostate cancer (mPC) at diagnosis is heterogeneous and unpredictable; thus alternative treatments such as immunotherapy are investigated. We retrospectively analyzed natural killer (NK) cells by flow cytometry in peripheral blood from $39 \mathrm{mPC}$ patients, with 5 year-follow-up, and their correlation with time to castration resistance (TCR) and overall survival (OS). In parallel, NK functionality was carried out against prostate tumor cell lines, analyzed for the expression of NK cell ligands, to identify the receptors involved in PC recognition. NK cells from patients with longer TCR and OS displayed high expression of activating receptors and high cytotoxicity. The activating receptors NKp30 and NKp46 were the most obvious predictive markers of OS and TCR in a larger cohort of $\mathrm{mPC}$ patients (OS: $p=0.0018$ and 0.0009 ; TCR: $p=0.007$ and < 0.0001 respectively, log-rank test). Importantly, blocking experiments revealed that NKp46, along with NKG2D and DNAM-1 and, to a lesser extent NKp30, were involved in prostate tumor recognition by NK cells. These results identify NK cells as potential predictive biomarkers to stratify patients who are likely to have longer castration response, and pave the way to explore therapies aimed at enhancing NK cells in mPC patients.

\section{INTRODUCTION}

Prostate cancer (PC) is the most common invasive cancer and the second and third leading cause of cancerrelated death among men in the U.S. and Europe respectively [1]. Localized tumors can be cured by surgical resection or radiotherapy. For metastatic castrate-sensitive PC (mCSPC) the recommended treatment is castration [2]. However castration resistance inevitably develops for all patients, thus resulting in a need for chemotherapeutic drugs and new treatments for metastatic castrate-resistant disease (mCRPC). Immunotherapy has emerged for over a decade as an alternative therapeutic approach for metastatic patients. The first currently only FDA-approved vaccine is a DC-based vaccine known as Sipuleucel-T (Provenge; Dendreon Seattle, WA, US) which increases survival for mCRPC [3]. Several other immunotherapies for advanced PC have progressed to phase II or phase III clinical trials : the GVAX-PCa (GM-CSF immunotherapy for cancer; Biosante Inc) cell-based cancer vaccine [4, 5], or the Ipilimumab (Yervoy, BMS Princeton, NJ), fully human anti-Cytotoxic T Lymphocyte Antigen-4 (CTLA4) monoclonal antibody, alone or in combination with radiotherapy $[6,7]$.

Metastatic PC at the time of diagnosis now represents less than $5 \%$ of PC cases [8]. The median 
survival of a man with metastases at the time of diagnosis has improved during the last decade from 30 to 49 months due to the PSA (prostate specific antigen) screening, which led to early detection of the disease, and because of new treatments at the time of CRPC [9]. Some prognostic factors have been determined for MPC: bone localizations (axial vs appendicular), visceral localizations, Gleason score $\geq 8$, performance status and PSA $\geq 65 \mathrm{ng} / \mathrm{ml}$ [10]. Despite these important advances, $\mathrm{mPC}$ at the time of diagnosis is still highly heterogeneous and unpredictable, with some patients long responders to sequential treatments, and others non responders. The challenge is now to improve risk stratification tools for this subgroup.

Natural killer (NK) cells belong to innate immune system and exert effector functions such as cytotoxic activity and cytokine production in antiviral and antitumor responses [11]. Human NK cells are defined as CD3CD56+ cells and represent $5 \%$ to $20 \%$ of circulating lymphocytes. According to membrane densities of CD56 and CD16, which mediates the antibodydependent-cellular cytotoxicity (ADCC), NK cells are classified into CD56 ${ }^{\mathrm{dim}} \mathrm{CD} 16+(90-95 \%$ of $\mathrm{NK}$ cells $)$ and CD56 ${ }^{\text {bright }}$ CD16- (5-10\%) subsets [12]. CD56 ${ }^{\text {bright }}$ cells are considered immature, rapidly proliferate, produce high levels of cytokines and have the ability to progressively differentiate into CD56 ${ }^{\mathrm{dim}}$ cells. In contrast, CD56 ${ }^{\mathrm{dim}}$ cells exert high cytotoxic potential. The effective function of NK cells depends on an intricate balance between activating and inhibitory receptors which are able to bind ligands present on target cells. Inhibitory receptors include the killer immunoglobulin-like receptors (KIR), CD94/ NKG2A, and ILT2/CD85j [13-17]. The main activating receptors of $\mathrm{NK}$ cells are the natural cytotoxicity receptors (NCRs: NKp46, NKp30, NKp44), NKG2D and DNAM1 (DNAX accessory molecule-1) [18, 19]. A hallmark of NK cell activation is degranulation, leading to CD107 externalization and release of lytic granule contents (perforin and granzyme) onto the surface of the target cell [20].

A 11-year follow-up pioneering study in human population reported that a low degree of $\mathrm{NK}$ cell cytotoxicity was correlated with increased cancer risk [21]. Since then, the prognostic value of NK cells has been explored : a low expression of NKp30 and NKp46 was shown to strictly correlate with enhanced progression of the disease in acute myeloid leukemia [22] and chronic lymphocytic leukemia [23]. There are now accumulating evidences for the role of NK cells in solid tumors; reduced expression of NCRs was associated with different forms of cancer such as melanoma [24-26], cervical cancer [27], breast cancer [28, 29], lung cancer [30-32], renal cell carcinoma [33] and gastrointestinal tumors (GIST) [34, 35]. However NK cells have been very scarcely studied in prostate cancer, excepted immunohistochemical (IHC) observations showing that the ligands for NKp30 and NKp46 are expressed on primary tumors and not on benign prostate hyperplasia [36]. A high count of CD56+ NK cells in prostate tumors after androgen deprivation therapy was associated with a good prognosis, and there was an inverse correlation between the density of CD56+ NK cells and seminal vesicle invasion [37]. Thus, NK cell activity may be a prognosis factor in PC patients.

Here, we analyze NK cell markers monitored exvivo in peripheral blood of patients with metastases at $\mathrm{PC}$ diagnosis and their correlation with clinical outcomes, i.e. time to castration resistance and overall survival. We also explore in-vitro the recognition mechanisms of prostate tumor cells by NK cells.

\section{RESULTS}

\section{Patient characteristics}

We conducted a retrospective study to analyze NK cells from a series of 39 patients with metastases at PC diagnosis including rare cases of patients with longterm survival and time to castration resistance (Table 1). Patients were observed for a median period of 62 months (range, 11 to 212 months). For first statistical analyses, patients were excluded if they were under bisphosphonates or corticosteroids at the time of blood sample. Patients were stratified into two groups according to the time to castration resistance, with an 18-months cutoff value: patients with long castration response (LCR), and patients with short castration response (SCR). The clinical characteristics (detailed for each patient in Supplementary Table 1) are summarized in Table 2. The patients selected for first analyses were sampled within two months after diagnosis $(n=18)$. To note, none of the following potential confounding factors: age at diagnosis, initial PSA, initial Gleason score, number and localization of metastases, were statistically different between LCR and SCR patients, even if the distribution of patients with 4 or more metastases, Gleason score $\geq 8$ and initial PSA $\geq 65 \mathrm{ng} /$ $\mathrm{ml}$ tended to be higher in the SCR compared to the LCR group. In this study, we analyzed two clinical endpoints: the overall survival (OS), measured from the diagnosis of metastases until the date of death or last follow-up; and the time to castration resistance (TCR), measured from the first day of castration until the date of castration resistance (Figure 1A).

\section{NK cells from LCR patients display high levels of activating receptors and high functionality}

Curves for OS and TCR were thus established for patients sampled at diagnosis $(n=18)$ (Figure 1B). The LCR $(n=8)$ and SCR $(n=10)$ groups were significantly discriminated according to log-rank test ( $p=0.00007$ for OS curves and $p<0.0001$ for TCR curves). NK cells 


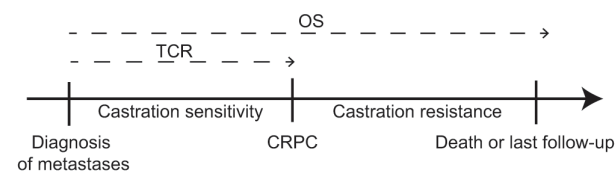

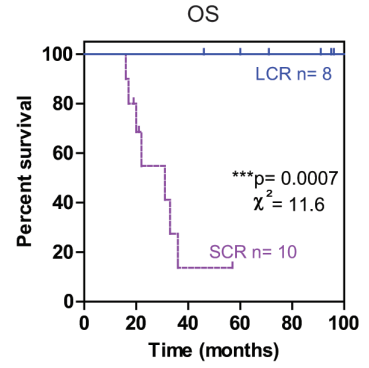

TCR

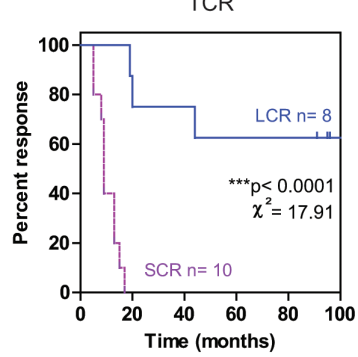

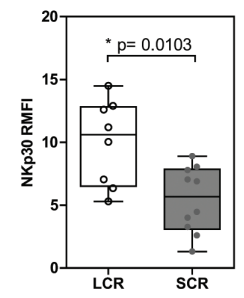

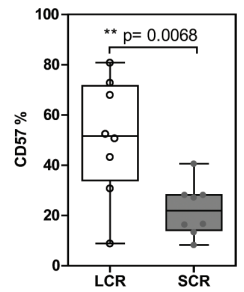

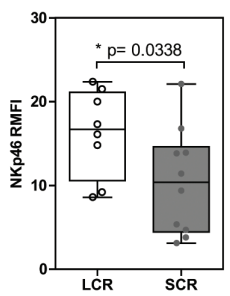
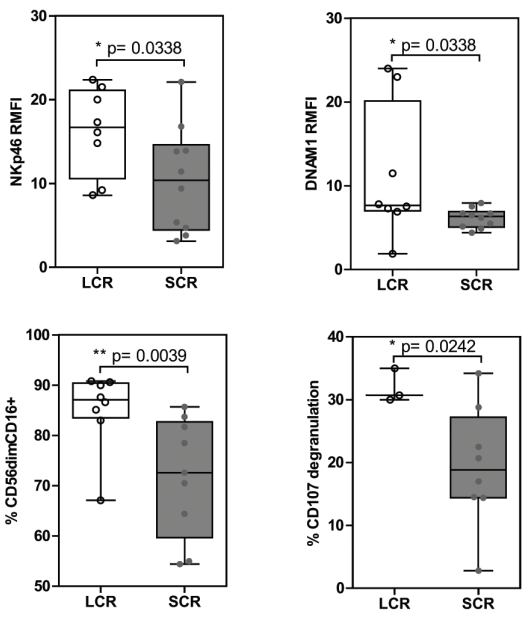

D
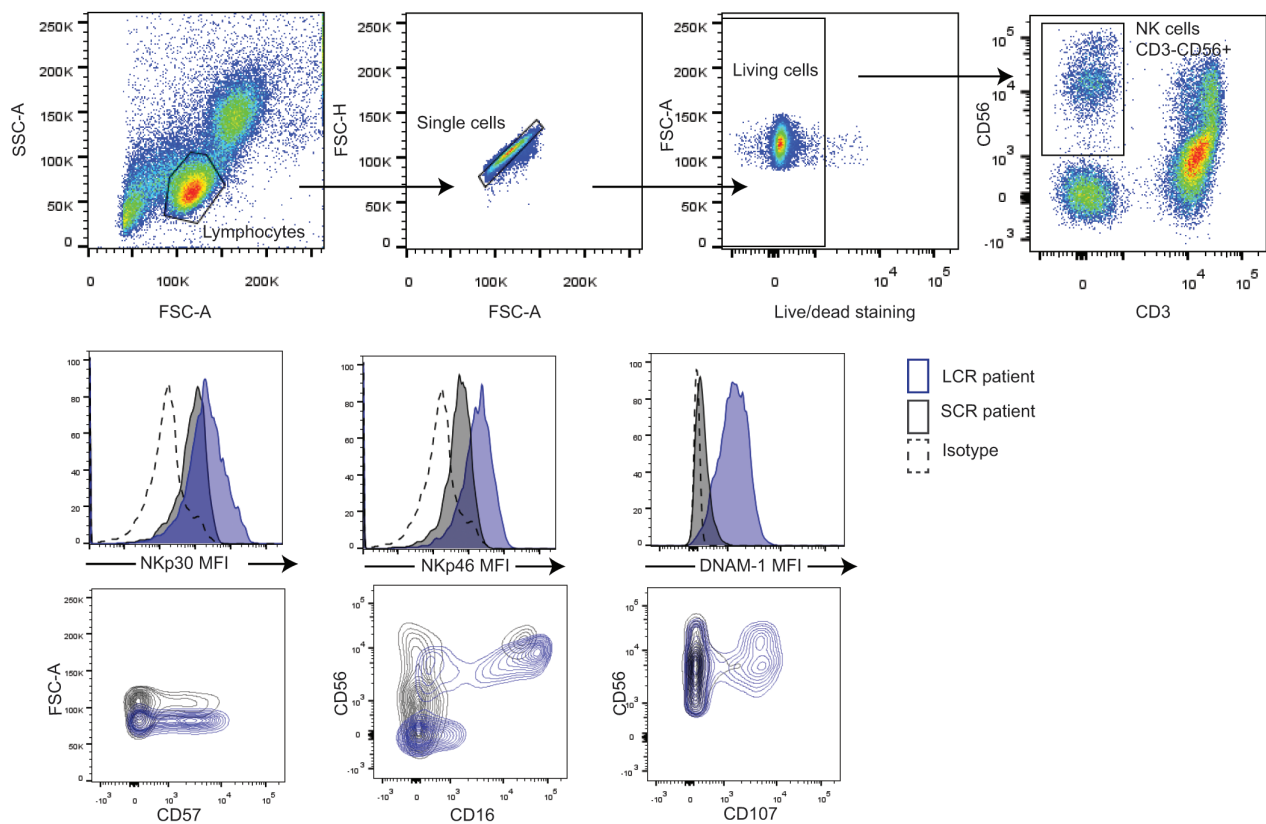

Figure 1: NK cells from mPC patients with longer survival and response to castration have strong cytotoxic potential. mPC patients sampled within 2 months after diagnosis of metastases and without treatment at sample $(n=18)$ were stratified into two groups, according to the time to castration resistance with an 18-months cutoff value: LCR (long castration response, $n=8$ ) and SCR (short castration response, $n=10$ ) patients. A. Clinical endpoints analyzed in this study: overall survival (OS) and time to castration resistance (TCR). B. Kaplan-Meier curves of OS and TCR. Blue solid line, LCR patients; pink dashed line, SCR patients. The relative differences in survival and response distribution $\left(\chi^{2}\right)$ and $p$ values were determined by log-rank statistics. C. The expression of NK cell markers on peripheral NK cells was analyzed by flow cytometry in LCR (white plots) and SCR (grey plots) patients sampled at diagnosis. The y axis shows the MFI ratio or the percentage of NK cells (CD56+CD3-) positive for each marker depending on uni- or bimodal expression. Data are represented by "box and whisker (min to max; horizontal lines represent mean values)" graphs. P values were obtained using MannWhitney test. $p<0.05=* ; p<0.01=* * ; p<0.001=* * *$. D. Gating strategy for NK cells (CD56+CD3- among living lymphocytes) and representative histogram or dot plot for each NK cell marker. 
Table 1. Cohort. Among patients with metastases at $\mathrm{PC}$ diagnosis $(\mathrm{n}=39)$, patients under treatment at the time of blood sample (corticosteroids or bisphosphonates) were initially excluded. Patients were divided according to the time to castration resistance: LCR pts (long castration response $>18 \mathrm{mo}$.) and SCR pts (short castration response $<18$ mo.). Selected patients for statistical analysis $(n=18)$ have been sampled within two months after diagnosis.

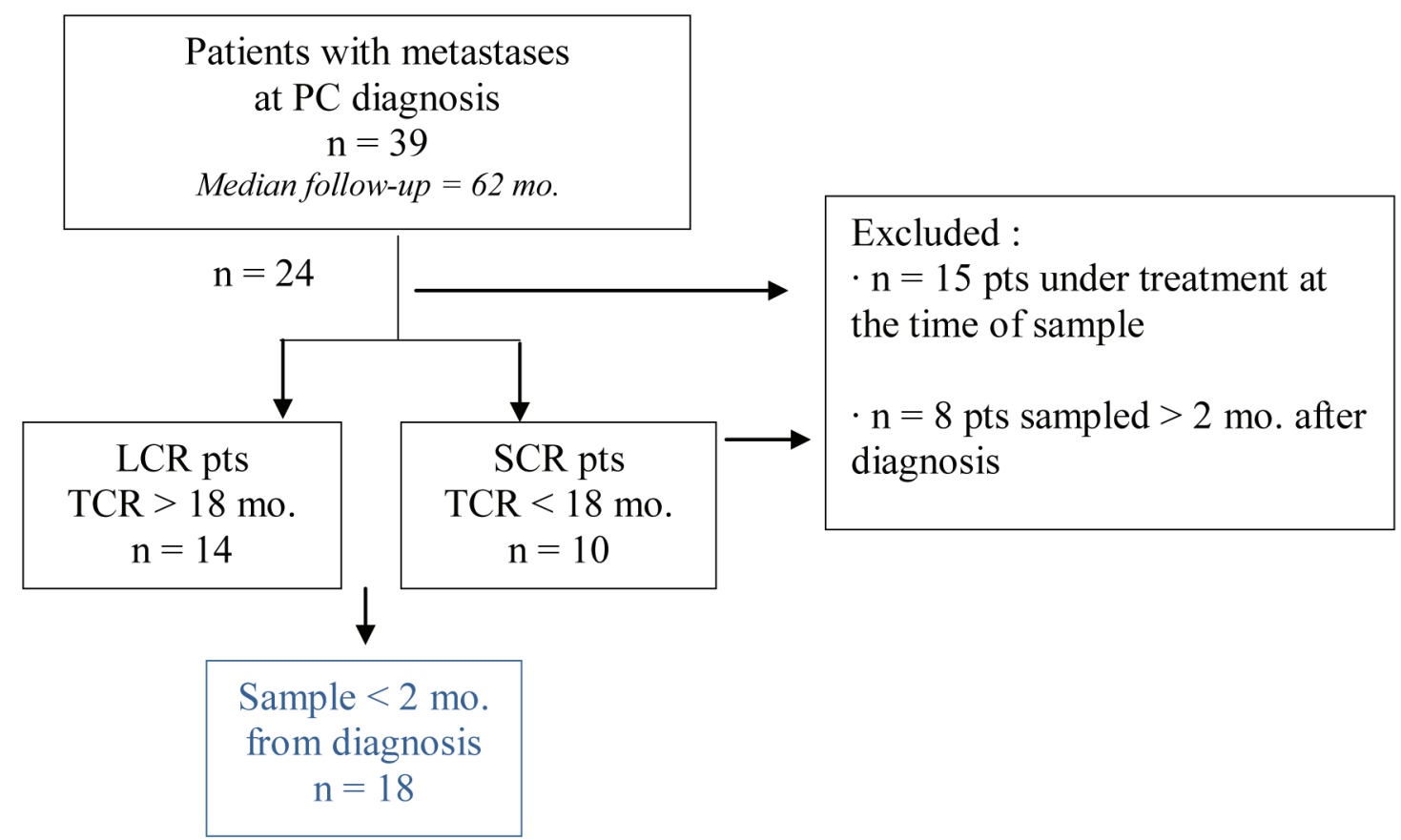

were isolated from peripheral blood sampled at diagnosis and were characterized ex-vivo by flow cytometry for the expression of the major NK cell receptors. Then, univariate analyses using Cox regression model was performed to determine if NK cell markers were significantly associated with OS and TCR. Cox regression analysis (values in Table 3) showed that NKp46, NKp30, DNAM-1, CD56dimCD16+ subset, CD57 and the degranulation marker CD107 were associated with OS (with $p$-values ranging from 0.009 to 0.20 ). The same markers were found significantly associated with TCR, with superior significant $p$-values (range, 0.002 to 0.10 ). All these factors have negative $\mathrm{z}$ coefficient and thus a positive effect on survival and response to castration.

Indeed, we observed that LCR patients (time to castration resistance $>18 \mathrm{mo}$.) expressed significantly higher levels of NK cell markers than SCR patients (time to castration resistance $<18 \mathrm{mo}$.) (Figure 1C, 1D): NKp30 ( $p=0.010$, median values of RMFI: 10.6 vs. 5.7 respectively), NKp46 ( $p=0.034$, RMFI median values 16.7 vs. 10.4$)$, DNAM-1 ( $p=0.034$, RMFI median values 7.7 vs. 6.3 ), CD57 ( $p=0.007$, median values of positive cells: $51.6 \%$ vs. $21.9 \%)$, CD56dimCD16+ $(p=$ 0.004 , median values $87.1 \%$ vs. $72.6 \%$, Mann-Whitney test). Moreover, NK cells from LCR patients degranulated with higher efficiency than NK cells from SCR patients in response to K562 target cell line ( $p=0.024$, median values
$30.7 \%$ vs. $18.9 \%$,). To note, CD107 degranulation assay was not available for all patients and thus was excluded in further analyses. Other NK cell markers were tested (i.e.; CD69, 2B4, ILT2, NKp44, CD94, NKG2D, \% of NK cells, CD16) and were not significantly linked with either OS or with TCR (Supplementary Figure 1A). Interestingly, NK cell markers but not $\mathrm{T}$ cell markers were significantly associated with longer survival and response to castration. Only CD69 and DNAM-1 were upregulated in T cells from LCR than SCR patients (Supplementary Figure 1B).

We compared the performance of NK cell markers with clinical parameters (Table 4). In this series of mPC patients, NK cell markers were more performant to discriminate LCR and SCR patients according to the time to castration resistance than the clinical parameters (AUC values for ROC curve; NK cell markers: range 0.76-0.87 and clinical parameters: range 0.51-0.76) (Table 4 and the ROC curves are available on Supplementary Figure 2). The Gleason score and the number of bone metastases were the most discriminant clinical parameters. The cutoff values for NK cell markers and clinical parameters were determined from the highest sensitivity value on the ROC curves. Spearman correlations between NK cell markers and clinical parameters are available in Supplementary Table 2. Particularly, NK cell markers were inversely correlated with the number of bone metastases, the presence of visceral metastases, Gleason score; which 
Table 2: Clinical characteristics of metastatic PC patients.

\begin{tabular}{|l|l|l|l|}
\hline Clinical parameter & LCR ( 18 mo.) & SCR (<18 mo.) & p-value \\
\hline $\mathrm{n}$ & 14 & 10 & \\
\hline Sample $<2$ mo. from diagnosis & 8 & 10 & \\
\hline Age at diagnosis, yrs (range) & $65(46-81)$ & $68(53-88)$ & $0.3194^{*}$ \\
\hline Initial PSA, ng/ml & $290 \pm 108$ & $150 \pm 53$ & $0.4606^{*}$ \\
\hline Initial Gleason Score (Min-Max) & $6-9$ & $7-9$ & $0.1982 \ddagger$ \\
\hline $\begin{array}{l}\text { No. of bone metastases : } \\
<4\end{array}$ & $5(36 \%)$ & $2(20 \%)$ & \\
$\geq 4$ & $4(29 \%)$ & $7(70 \%)$ & $0.2480 \ddagger$ \\
None & $2(14 \%)$ & $1(10 \%)$ & \\
Unknown & $3(21 \%)$ & $0(0 \%)$ & \\
\hline Appendicular bone metastases : & $5(36 \%)$ & $6(60 \%$ & \\
Yes & $7(50 \%)$ & $4(40 \%)$ & $0.5802 \ddagger$ \\
No & $2(14 \%)$ & $0(0 \%)$ & \\
Unknown & $10(72 \%)$ & $9(90 \%)$ & \\
\hline Axial bone metastases : & $3(21 \%)$ & $1(10 \%)$ & $0.7688 \ddagger$ \\
Yes & $1(7 \%)$ & $0(0 \%)$ & \\
No & & & \\
Unknown & $2(14 \%)$ & $4(40 \%)$ & \\
\hline Visceral metastases : & $8(57 \%)$ & $4(40 \%)$ & $0.5507 \ddagger$ \\
Yes & $4(29 \%)$ & $2(20 \%)$ & \\
No & $2(14 \%)$ & $6(60 \%)$ & \\
Unknown & $8(57 \%)$ & $3(30 \%)$ & $0.0742 \ddagger$ \\
\hline Lymph node metastases : & $4(29 \%)$ & $1(10 \%)$ & \\
Yes & $112.1 \pm 12.7$ & $27.2 \pm 3.9$ & $<0.0001^{*}$ \\
No & $73.5 \pm 13.5$ & $10.3 \pm 1.3$ & $<0.0001^{*}$ \\
\hline Unknown & & & \\
\hline Median OS, mo. & & & \\
\hline Median TCR, mo. & & & \\
\hline
\end{tabular}

LCR, long castration response; SCR, short castration response; PSA, prostate specific antigen; OS, overall survival; TCR, time to castration resistance.

Data are indicated as median (range) for age at diagnosis, median (SD) for initial PSA, OS and TCR, $\mathrm{n}$ ( $\%$ of patients) for number and presence of metastases.

* p-value obtained using Mann-Whitney test

₹ p-value obtained using Fisher test

Table 3: Cox regression analyses of NK cell markers on OS and TCR curves.

\begin{tabular}{|l|c|c|c|c|c|c|c|c|}
\hline & \multicolumn{4}{|c|}{ OS } & \multicolumn{4}{c|}{ TCR } \\
\hline Marker & HR & $\mathbf{9 5 \%}$ CI & z coeff & p-value & HR & 95\% CI & z coeff & p-value \\
\hline NKp46 & 0.76 & 0.61 to 0.94 & -2.52 & 0.012 & 0.83 & 0.74 to 0.93 & -3.05 & 0.002 \\
\hline \% CD56dimCD16+ & 0.90 & 0.84 to 0.97 & -2.60 & 0.009 & 0.93 & 0.88 to 0.97 & -2.88 & 0.003 \\
\hline NKp30 & 0.73 & 0.54 to 0.98 & -2.10 & 0.035 & 0.75 & 0.61 to 0.93 & -2.58 & 0.009 \\
\hline \% CD107 & 0.89 & 0.81 to 0.99 & -2.18 & 0.028 & 0.92 & 0.86 to 0.99 & -2.01 & 0.044 \\
\hline CD57 & 0.95 & 0.89 to 1 & -1.65 & 0.098 & 0.97 & 0.94 to 1 & -1.72 & 0.084 \\
\hline DNAM-1 & 0.84 & 0.65 to 1.09 & -1.27 & 0.203 & 0.82 & 0.64 to 1.04 & -1.61 & 0.105 \\
\hline
\end{tabular}

OS, overall survival; TCR, time to castration resistance; HR, hazard ratio; 95\% CI, 95\% confidence interval; z coeff, covariate coefficient (indicates the direction and degree of flexing that the predictor has on the survival curve); p-value, significance level 
Table 4: Comparison of ROC curve values for NK cell markers and clinical parameters.

\begin{tabular}{|c|c|c|c|c|c|}
\hline Test & $\mathbf{A U C}$ & p-value & \begin{tabular}{|l|} 
Sensitivity \\
$(95 \%$ CI $)$
\end{tabular} & $\begin{array}{l}\begin{array}{l}\text { Specificity } \\
(95 \% \mathrm{CI})\end{array} \\
\end{array}$ & $\begin{array}{r}\text { Cut-off } \\
\text { value } \\
\end{array}$ \\
\hline NKp30 & 0.82 & 0.020 & $75 \%$ & $60 \%$ & 6.97 \\
\hline NKp46 & 0.76 & 0.062 & $75 \%$ & $80 \%$ & 14.35 \\
\hline DNAM-1 & 0.79 & 0.041 & $87.5 \%$ & $80 \%$ & 6.81 \\
\hline$\%$ CD57 & 0.87 & 0.011 & $87.5 \%$ & $87.5 \%$ & 29.5 \\
\hline$\%$ CD56dimCD16+ & 0.87 & 0.009 & $87.5 \%$ & $77.8 \%$ & 82.35 \\
\hline Gleason score & 0.76 & 0.080 & $71.4 \%$ & $77.8 \%$ & $\geq 8$ \\
\hline Initial PSA & 0.56 & 0.624 & $53.8 \%$ & $58.3 \%$ & $\geq 65 \mathrm{ng} / \mathrm{ml}$ \\
\hline No. of bone metastases & 0.71 & 0.143 & $71.4 \%$ & $70 \%$ & $\geq 4$ \\
\hline Appendicular bone metastases & 0.57 & 0.594 & $62.5 \%$ & $60 \%$ & Yes/ No \\
\hline Axial bone metastases & 0.52 & 0.894 & $25 \%$ & $90 \%$ & Yes/ No \\
\hline Visceral metastases & 0.57 & 0.594 & $62.5 \%$ & $60 \%$ & Yes/ No \\
\hline Lymph node metastases & 0.59 & 0.505 & $62.5 \%$ & $70 \%$ & Yes/ No \\
\hline
\end{tabular}

AUC, area under the curve; PSA, prostate specific antigen.

The cut-off values for NK cell markers and clinical parameters were obtained from the highest sensitivity value. Cutoff values are expressed as RMFI for NKp30, NKp46 and DNAM-1; as percentage of NK cells positive for CD57 and CD56 ${ }^{\mathrm{dim}} \mathrm{CD} 16+$.

A

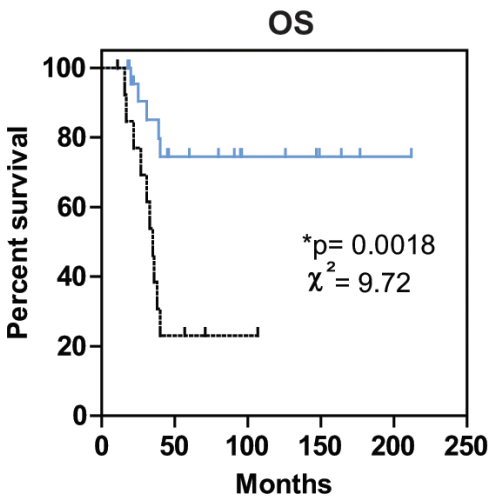

B

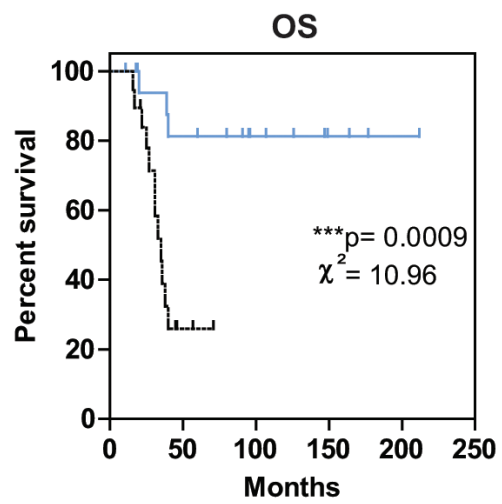

NKp30

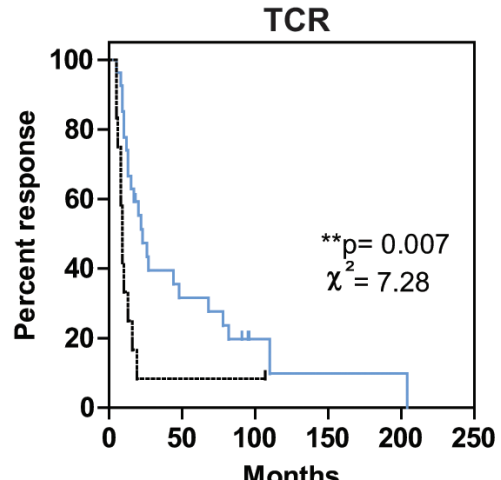

1 NKp30 high $(n=25)$

-..-- NKp30 low $(n=14)$

$n=39$

NKp46

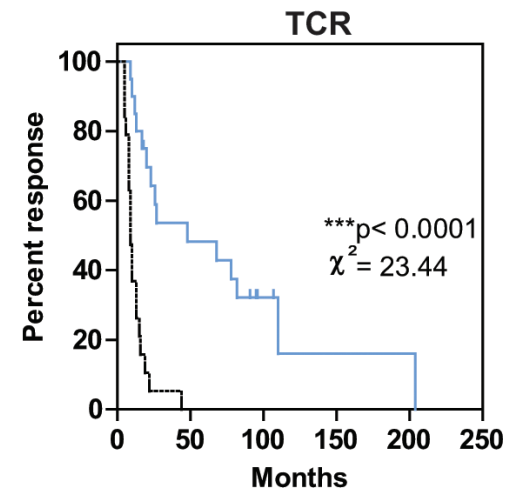

Figure 2: NKp46high and/or NKp30high phenotype is associated with good prognosis in mPC patients. Kaplan-Meier curves for OS and TCR were performed on data from the total cohort of $39 \mathrm{mPC}$ patients $(n=18$ patients used for first statistical analyses and $n=21$ patients initially excluded because under treatment at sample or sampled at distance from diagnosis). Patients were stratified according to the previously defined cut-off value of NKp30 A. and NKp46 B.. Log-rank statistics were used to compare high (solid line) and low (dashed line) curves; $\chi^{2}$ and $p$ values are indicated on each graph with $p<0.05=* ; p<0.01=* * ; p<0.001=* * *$. $n$ indicates the number of corresponding patients in each arm. 
are poor prognosis factors in PC, and positively associated with the presence of axial bone metastases, which is a good prognosis indicator.

We show that NK cells from $\mathrm{mPC}$ patients with longer response to castration display efficient phenotypic and functional pattern associated with high expression of activating receptors and molecules involved in $\mathrm{NK}$ cell maturation and degranulation. NK cell markers are inversely correlated with prognostic factors associated with poor prognosis.

\section{NKp30 ${ }^{\text {high }}$ and NKp46 ${ }^{\text {high }}$ phenotype predict good prognosis}

We determined different subgroups based on NK cell marker expression : patients were stratified into high and low subgroups according to cut-off values of NK cell markers obtained in table 4, from the highest sensitivity value on the ROC curves. To obtain a sufficient number of patients for potent statistical analyses, in addition to the 18 patients used for first statistical analyses, we added patients initially excluded because under treatment at the time of blood sample $(n=15)$ or sampled at distance from diagnosis of metastases $(n=6)$. Kaplan-Meier curves for OS and TCR revealed that NKp30 and NKp46 expression significantly predicted $\mathrm{OS}$ and TCR in these $\mathrm{mPC}$ patients. We first analyzed patient survival and response to castration according to NKp30 expression (Figure 2A). Probability of survival at 3 years was $85 \%$ for patients with $\mathrm{NKp} 30^{\text {high }}$ and $38 \%$ in patients with $\mathrm{NKp} 30^{\text {low }}$ expression $(p=0.0018)$. Probability of response to castration at 3 years was $39 \%$ for patients with NKp30 $0^{\text {high }}$ and $8 \%$ for the patients with NKp30 low expression $(p=$ $0.007)$. We then analyzed patient survival and response to castration according to NKp46 expression (Figure 2B). The expression of NKp46, high or low, was also predictive of OS and TCR (3-year OS $=94 \%$ vs. 39\% respectively; $p=0.0009 ; 3$-year TCR $=54 \%$ and $5 \%$ respectively; $p$ $<0.0001)$. The association of CD57, CD56 ${ }^{\mathrm{dim}} \mathrm{CD} 16+$ and CD107 subgroups with survival and response to castration was lower (values on Supplementary Table 3 ). To note, NKp30 and NKp46 remained highly predictive of OS and TCR if log-rank rest was performed only on the smallest cohort of 21 additional patients (see Supplementary Table 3).

\section{NK cells markers involved in the NK-mediated lysis of prostate tumor cells}

Because NK cell functions are dependent on the expression of ligands at the surface of the target cells, the ligands for NK cell receptors were analyzed by flow cytometry in PC3, LNCaP and DU145 prostate tumor cell lines derived from metastatic prostate adenocarcinoma (Figure 3A). HLA class-I molecules were expressed on all cell lines. The prostate tumor cell lines expressed high levels of MICA/B and ULBP1/2/3, which are ligands for the activating receptor NKG2D, and these observations were confirmed through detection with NKG2D-Fc recombinant protein. Furthermore, Nectin-2 and CD155/ PVR which are ligands for the activating adhesion receptor DNAM-1, were highly expressed in PC3, LNCaP and DU145. In contrast, prostate cancer cell lines displayed lower levels of NKp30-L and NKp46-L, even if detection with Fc-molecules has lower binding affinity than specific mAbs.

To determine the receptors involved in prostate tumor cell recognition and their contribution, IL-2/IL-15activated PBMCs from healthy donors were pre-incubated with blocking antibodies against each NK cell receptor, and then used in CD107 degranulation assay against prostate cell lines or K562 as positive control. The results of the blocking experiments are expressed as percentage of modulation of NK cell degranulation and are summarized in Figure 3B. In accordance with the literature, NKp30, DNAM-1, NKG2D and, to a lesser extent, NKp46 were the receptors involved in K562 cell line recognition by NK cells [38-42]. Indeed, blockage of these receptors inhibited NK cell degranulation against K562. Regarding prostate tumor cell lines, we found that masking NKp46 significantly decreased the degranulation of $\mathrm{NK}$ cells against PC3 (56\%), LNCaP (55\%) and DU145 (64\%), with significant higher level than for K562 cell line $(p<$ 0.01). Anti-DNAM-1 and anti-NKG2D antibodies also decreased NK cell degranulation against PC3 (39\% and $45 \%$ respectively), LNCaP (44\% and 56\%) and DU145 (47\% and 43\%). Blocking NKp30 decreased modestly NK cell degranulation against PC3 (13\%), LNCaP (13\%) and DU145 (0.8\%) in agreements with the lower expression of NKp30 ligand(s) observed in Figure 3A. The raw data expressed as percentage of CD107ab in presence or absence of blocking $\mathrm{mAbs}$ are depicted in Supplementary Figure 3.

Thus, these results identified NKp46, DNAM-1, NKG2D and, to a lesser extent, NKp30 as the receptors regulating the recognition of prostate tumor cells by activated NK cells.

\section{DISCUSSION}

Patients with metastatic prostate cancer at the time of diagnosis have a heterogeneous and unpredictable prognosis, with some patients long responders to sequential treatments, and others non responders. We provide original findings about the correlation of peripheral NK cell phenotype and clinical outcomes in these patients. NK cells from patients with longer survival and time to castration resistance display high expression of NK cell receptors, notably NKp46 and NKp30, which we identify here as involved in prostate tumor cell recognition by NK cells. Hence, the functional experiments 
corroborate the ex vivo data on the identification of the NK cell receptors involved on the control of metastatic PC. Peripheral NK cells from LCR patients display high expression of molecules involved in NK cell cytotoxicity (NKp30, NKp46), costimulation (DNAM-1), maturation (CD57) and degranulation (CD107). To note, higher levels of CD16 in LCR patients suggest a high ability to perform ADCC functions; and this could be exploited to improve the efficacy of therapeutic Ab-mediated effects.
Previous studies in the literature indicate that prostate cancer cells express ligands that could drive NK cell activation. Indeed, exosomes produced by human PC cells express ligands for NKG2D on their surface and induced down-regulation of NKG2D on NK cells [43]. Here, we screened a large panel of NK cell ligands on prostate cancer cell lines and we identified which NK cell receptors are involved in $\mathrm{PC}$ recognition. The prostate tumor cells express ligands for the major activating NK

A

\begin{tabular}{|c|l|c|c|c|c|}
\hline & & \multicolumn{3}{|c|}{ Prostate cell line } & \\
\hline & Ligand & PC3 & LNCaP & DU145 & \\
\hline \multirow{2}{*}{ DNAM-1-L } & Nectin-2 & +++ & +++ & +++ & \multirow{2}{*}{+++} \\
\cline { 2 - 5 } & CD155 PVR & +++ & +++ & ++++ & \\
\hline \multirow{3}{*}{ HLA family } & HLA-ABC & +++ & ++ & ++++ & \multirow{3}{*}{++} \\
\cline { 2 - 5 } & HLA-E & ++ & ++ & +++ & \\
\cline { 2 - 5 } & HLA-G & - & + & - & \\
\hline & MICA/B & +++ & ++ & +++ & \\
\cline { 2 - 5 } & ULBP1 & ++ & ++ & +++ & \multirow{3}{*}{+++} \\
\cline { 2 - 5 } & ULBP2 & ++ & ++ & + & \\
\cline { 2 - 5 } & ULBP3 & ++ & + & - & \\
\cline { 2 - 5 } & NKG2D-Fc & ++++ & ++++ & +++ & \\
\hline NKp30-L & NKp30-Fc & + & + & \pm & + \\
\hline NKp46-L & NKp46-Fc & + & + & + & + \\
\hline
\end{tabular}

NOTE: The scores indicate: -: no detectable expression, \pm : less than $5 \%$; $+: 5 \%$ to $10 \%$; $++: 10 \%$ to $50 \%$; $+++: 50 \%$ to $100 \%$; $++++: 100 \%$ of cells express the ligand.

B
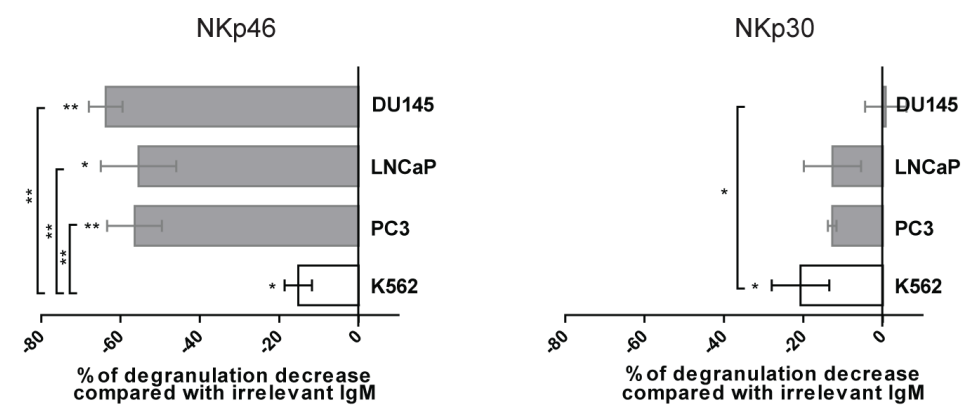

DNAM-1

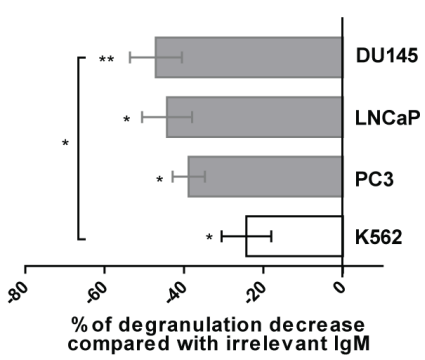

NKG2D

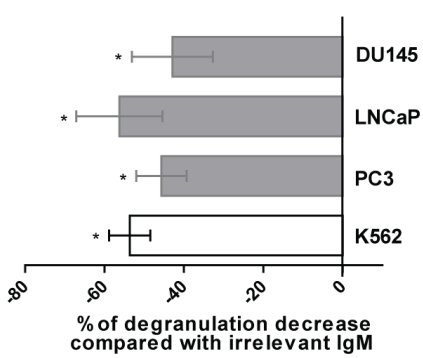

Figure 3: Receptors involved in human prostate cancer cell recognition by NK cells. A. Expression of NK cell ligands on PC3, DU145 and LNCaP prostate cell lines by flow cytometry. The score indicate: - : no detectable expression; \pm : less than 5\%; + : $5 \%$ to $10 \%$; ++ : $10 \%$ to $50 \%$; +++ : $50 \%$ to $100 \%$; ++++ : $100 \%$ of cells express the ligand. The receptor matching with each ligand is indicated. B. PBMCs from healthy donors were activated overnight in IL-2 and IL-15, and used in a 4-hours CD107 degranulation assay against PC3, DU145 or LNCaP prostate tumor cell lines, in the presence of blocking antibodies for NKp46, NKp30, DNAM-1, NKG2D or irrelevant isotype control mAb. E:T = 10:1. The percentage of CD107 degranulation by NK cells was evaluated by flow cytometry. The decreased effect of the respective blocking antibody compared with the irrelevant isotype control mAbs was evaluated with a nonparametric Wilcoxon test. The difference between prostate cell lines and K562 cell line was evaluated with Mann-Whitney test. Results are representative of 4 independent experiments. $p<0.05=* ; p<0.01=* * ; p<0.001=* * *$. 
cell receptors, in particular high levels of ligands for DNAM-1 and NKG2D, underlying the role of innate immunity in tumor control. We identified NKp46 as the crucial receptor involved in prostate cancer recognition by normal NK cells, along with DNAM-1 and NKG2D, and NKp30 to a lesser extent. Such a role for NKp46 was previously suggested when Arnon et al. showed by IHC that primary human prostate tumors and not benign prostate hyperplasia expressed ligands for NKp46 and NKp30 [36]. Interestingly, our observations also emphasize that the NK cell receptors involved in NK cell antitumor immunity differ according to the tumor type: in breast cancer the main receptors involved in NK cell cytotoxicity are DNAM-1 and NKG2D [29] ; whereas in melanoma NKp30, NKp44 and NKG2D are the receptors that trigger NK cell functions [24]. The recognition tightly depends on the repertoire of ligands expressed by the tumor cells, but also the level of receptors expressed on NK cells. Thus, downregulation of the ligand might be an escape mechanism of the tumor to evade NK cell cytotoxicity, and inversely our results suggest that upregulation of the NK cell receptors- notably NKp46, in some long-responders patients, could favor tumor control in prostate cancer.

Which are the mechanisms that could explain the heterogeneity of NK cell markers observed in metastatic PC patients? NKp46 is frequently deregulated $[27,28]$ and involved in tumor editing [44]. NKp30 recognizes the tumor antigens B7-H6 and BAG6 (also known as BAT3) but only B7-H6 has been involved in tumor clearance due to its restricted expression on malignant cell lines [38]. Several mechanisms could explain the downregulation of NCRs in some patients: chronic ligands exposure as it has been described for DNAM-1 or NKG2D [29, 45]; or cytokines released by the tumor, such as TGF- $\beta 1$ which has been shown to downregulate NKp30 and NKG2D $[46,47]$. Screening of immunosuppressive factors such as TGF- $\beta 1$ in the plasma of metastatic PC patients will further be investigated. Recently, elevated levels of cytokines involved in inflammation (IL-8, TNF- $\alpha$, MCP1) have been associated with poorer PC outcome [48]. In line with these results, impaired NK cell activity analyzed through IFN- $\gamma$ levels has been recently associated with prostate cancer progression [49]. Together with our study, these observations strengthen the importance of immune context in PC.

We show that NKp30 and NKp46 are the most predictive markers for OS and TCR in a larger cohort that included patients under treatment or sampled at distance from diagnosis. Interestingly, NKp30 and NKp46 were also the receptors associated with increased survival in AML patients [22]. NKp46 was shown to control melanoma metastases [50] and tumor growth of lymphoma tumors [51] in mouse models and our study emphasizes its role in controlling human metastatic prostate cancer. Distinct NKp30 splice variants have been characterized, demonstrating opposite functional effects. The predominant expression of the immunosuppressive $\mathrm{NKp30c}$ isoform was associated with poor prognosis in GIST patients [35]. This could provide additional information to explore whether the relative expression of the different isoforms of NKp30 and/or NKp46 is associated with prostate cancer outcome.

What is the relevance of NK cell markers compared to current prognostic factors? New prognostic factors for metastatic PC have been identified: visceral metastases represent negative prognostic factors and are associated with aggressive disease [52]; in contrast, axial bone metastases are associated with better survival than appendicular metastases [53]. Here, NKp30 and NKp46 meet these emerging prognostic factors: inverse correlation with poor prognostic factor such as visceral metastases and positive correlation with good prognosis factor such as axial bone metastases. To note, the cut-off values that we obtained for clinical parameters were concordant with those described previously in the literature [10].

The NK cell infiltrate is often associated with a lower risk of relapse and/or longer survival: NK cell infiltrate was associated with regression in melanocytic lesions [54], predicted progression-free survival in localized GIST [34], and was associated with a good prognosis in prostate tumors after castration [37]. The NKp30 splice variants determined the prognosis of patients with GIST [35]. Interestingly, we show here that the prognostic value of NK cells is suitable in peripheral blood even in a solid tumor; therefore NK cell markers could be screened on blood samples, easily accessible for routine diagnosis, thus erasing the difficulty to access intratumoral lymphocytes. This suggests that peripheral NK cells may reflect the intratumoral phenotype and would represent tumorinduced subsets as already reported in breast cancer [55]. Together, these data strongly encourage the validation of immune biomarkers for optimal stratification of patients with solid tumors.

The correlation of efficient NK cells in patients with longer time to castration resistance and survival is a strong argument for their involvement in prostate cancer. Thus, our findings strongly suggest that enhancing NK cell efficiency could provide a promising immunotherapeutic approach for metastatic PC; and this work pave the way for further prospective and validation studies. Furthermore, screening for NK cell profiles in peripheral blood provides a non-invasive, easily measurable prognostic biological parameter, and could therefore be useful to improve risk stratification and to design better tailored treatment strategies in metastatic PC patients. 


\section{PATIENTS AND METHODS}

\section{Patients characteristics}

Patients with metastatic disease at the time of PC diagnosis were recruited in the period from December 1995 to January 2012 in a comprehensive cancer center (Institut Paoli-Calmettes, Marseille, France). The project was reviewed by the internal review board of Institut Paoli-Calmettes and the study was approved by a central national ethics committee. All patients provided written informed consent. 39 patients with metastatic PC at diagnosis were recruited during this period. To ensure an unbiased analysis, patients were excluded if they were under chemotherapy or radiotherapy at the time of blood sample because of possible effects on immune cells. Patients were stratified into two groups according to the time to castration resistance, with an 18-months cutoff value: patients with long castration response (LCR, median: 73.5 months (mo.) and median OS 112.1 mo.), and patients with short castration response (SCR, median $=10.3$ mo. and median OS $27.2 \mathrm{mo}$.).

The following clinical variables were retrospectively collected: age, initial PSA, initial Gleason Score, number and localization of bone metastases (axial and appendicular), presence of visceral (lung and/or liver) and lymph node metastases, serum levels of: alkaline phosphatase (ALP), lactate dehydrogenase (LDH), hemoglobin, performance status, previous treatments and treatments at the day of blood sample, follow-up (diagnosis of metastases, start of castration, castration resistance diagnosis, death or last follow-up). Clinical data where more than $30 \%$ of values per group were missing were excluded from further analyses (ALP, LDH, hemoglobin and performance status). Clinical characteristics are summarized in Table 2, and detailed along with the treatments received for each patient during their follow-up in Supplementary Table 1.

\section{PBMC isolation from blood samples}

Peripheral blood mononuclear cells (PBMC) were purified from blood samples by Lymphoprep (Lymphocyte separation medium, Eurobio, France) density gradient centrifugation. The cells from peripheral blood were washed and resuspended in RPMI (Roswell Park Memorial Institute, Gibco Life Technologies) medium and then frozen before phenotype and functional analysis.

\section{Flow cytometry analyses}

Peripheral NK cells were characterized ex vivo by multicolor flow cytometry analyses. Thawing cells were incubated with conjugated antibodies or isotypic controls for 30 minutes at $4^{\circ} \mathrm{C}$, washed and extemporaneously analyzed on LSRFortessa cytometer (BD Biosciences). NK cells (gated on CD3-CD56+ in lymphocyte FSC/SSC parameters, followed by elimination of the doublets based on FSC-A/FSC-H parameters and removal of dead cells using a cell viability marker, Live/Dead Aquadead kit (Invitrogen, Life Technologies, Grand Island, NY)) were labeled with PE (Phycoerythrin)- conjugated antihuman mAbs: NKp44 (clone Z231), NKp30 (clone Z-25), NKp46 (clone BAB281), 2B4 (clone C1.7), NKG2D (clone ON72), CD94 (clone HP-3B1), CD69 (clone TP1.55.3), ILT2 (CD85j, clone HP-F1; Beckman Coulter, Miami, FL), DNAM-1 (CD226, clone F22; BD Pharmingen, San Jose, CA), CD57 (clone HCD57; Biolegend, San Diego, $\mathrm{CA}$ ), and allophycocyanin-Cy7 (APC-Cy7)- CD16 (clone 3G8). Data analysis was done using FACSDiva (BD Biosciences) or FlowJo (TreeStar Inc.) programs. The phenotypic data in the paper are represented as the percentage of positive cells or the expression level (RMFI for ratio MFI; ratio between the specific and control isotype staining) depending on the marker.

Prostate tumor cell lines were labeled with HLAABC-FITC (clone B9.12.1, Beckman Coulter), HLAE-PE (clone 3D12HLA-E, eBioscience), HLA-G-FITC (clone MEM-G/9, Abcam), MICA/B-PE (clone 6D4, BD Pharmingen), ULBP1-PE (clone 170818), ULBP2PE (clone 165903), ULBP3-PE (clone 166510, R\&D Systems), Nectin-2-PE (clone R2.477.1, Beckman Coulter), CD155-PE (clone SKII.4, Biolegend). NKp30Fc, NKG2D-Fc and NKp46-Fc (R\&D Systems) chimeric proteins were used to detect ligands for NKp30, NKG2D and NKp46 respectively.

\section{Cell lines}

PC3, DU145 and LNCaP cell lines are derived from metastatic prostate cancer samples, castrateresistant for PC3 and DU145; and castrate-sensitive for LNCaP. Cell lines were purchased from American Type Culture Collection (ATCC). PC3 was cultured in Dulbecco's modified Eagle's medium (DMEM, Gibco Life Technologies) supplemented with $10 \%$ fetal calf serum (FCS, Lonza), and DU145 and LNCaP were cultured in RPMI supplemented with $10 \%$ FCS. K562 cell line is derived from human leukemia cell line (ATCC) and was cultured in RPMI supplemented with 10\% FCS.

\section{CD107 degranulation assay}

To evaluate the functionality of NK cells from PC patients, PBMCs from patients were activated overnight in IL-2 (100 U/ml, Chrion) and IL-15 (20 ng/ml; Miltenyi Biotec), and incubated with K562 cell line at effector : target (E:T) ratio of $10: 1$ during 4 hours at $37^{\circ} \mathrm{C}$, 
with monensin (Golgi Stop, BD Biosciences), FITCconjugated anti-CD107a (LAMP1) and FITC-conjugated anti-CD107b (LAMP2) mAbs. Cells were then washed in PBS and stained for 30 minutes at $4^{\circ} \mathrm{C}$ with PerCPCy5.5-conjugated anti-CD3 and $\mathrm{PC} 7$-conjugated antiCD56 antibodies. The percentage of CD3-CD56+ NK cells positive for CD107 was analyzed on a LSRFortessa cytometer (BD Biosciences).

For blocking experiments to determine which receptors are involved in prostate tumor cell recognition by NK cells, PBMCs from healthy donors were preincubated 30 min with blocking mAbs or saturating concentrations of appropriate isotype controls. Blocking mAbs directed against NK cell receptors were produced in Pr Moretta's laboratory: anti-NKp30 (F252), anti-NKp46 (KL247), anti-DNAM (F5), anti-NKG2D (ON72). Then, prostate tumor cell lines or K562 cell line as positive control were added at effector : target (E:T) ratio of 10:1 followed by the same protocol as above.

\section{Methodology and statistical analyses}

All statistical analyses were performed using Prism software (GraphPad), except Cox regression with R 3.01 software. Correlations between patient groups and clinical features were analyzed using the Mann-Whitney test or the Fisher's exact test (variable with two groups). Overall survival (OS) was measured from the date of diagnosis of metastases until the date of death or the last followup. Time to castration resistance (TCR) was measured from the first day of castration until the date of castration resistance. OS and TCR were estimated using the KaplanMeier method and curves were compared with the log-rank test. Univariate Cox proportional hazard regressions were performed with R software to analyze the effect of several risk factors on OS and TCR curves. Receiver operating characteristic (ROC) curve analysis was used to assess the discrimination performance of biological and clinical parameters, and to identify the optimal cutoff value. The Mann-Whitney test was used to compare two groups of data (LCR vs SCR patients) and the Wilcoxon test was used to compare paired data in functional experiments with blocking antibodies. Statistical significance was accepted at the $5 \%$ level with no adjustment for multiple testing.

\section{ACKNOWLEDGMENTS}

The authors thank Philippe Livrati and Sylvaine Just-Landi for excellent technical assistance; the Departments of Surgical Oncology, Pathology and the Outpatient Unit at the Institut Paoli-Calmettes as well as the patients for their contribution to this work; and Pr. Norbert Vey from the department of Hematology at the Institut Paoli-Calmettes for critical reading of the manuscript and helpful comments. This work was supported by grants from the French Cancer Institute (INCa, PAIR prostate program \#R10111AA) and SIRIC (INCa-DGOS-Inserm 6038 grant). The team of Pr Daniel Olive is labeled "Equipe FRM DEQ 201 40329534" " and DO is Senior Scholar of Institut Universitaire de France. CP was funded by the Institut National du Cancer for 2 years. AM was supported by Associazione Italiana Ricerca per la Ricerca sul Cancro (AIRC)-Special Project 5x1000 no. 9962 and IG 2014 Id. 15704.

\section{DISCLOSURE OF POTENTIAL CONFLICTS OF INTEREST}

$\mathrm{AM}$ is a founder and shareholder of Innate-Pharma (Marseille, France). The remaining authors have no conflicting financial interests.

\section{Abbreviations}

CSPC, castrate-sensitive prostate cancer; CRPC, castrate-resistant prostate cancer; GS, gleason score; $\mathrm{mPC}$, metastatic prostate cancer; NCRs, natural cytotoxicity receptors; NK, natural killer; OS, overall survival; PSA, prostate specific antigen; TCR, time to castration resistance

\section{REFERENCES}

1. Ferlay J, Steliarova-Foucher E, Lortet-Tieulent J, Rosso S, Coebergh JW, Comber H, Forman D and Bray F. Cancer incidence and mortality patterns in Europe: estimates for 40 countries in 2012. Eur J Cancer. 2013; 49:1374-1403.

2. MacVicar GR and Hussain MH. Emerging therapies in metastatic castration-sensitive and castration-resistant prostate cancer. Curr Opin Oncol. 2013; 25:252-260.

3. Kantoff PW, Higano CS, Shore ND, Berger ER, Small EJ, Penson DF, Redfern CH, Ferrari AC, Dreicer R, Sims RB, $\mathrm{Xu}$ Y, Frohlich MW and Schellhammer PF. Sipuleucel-T immunotherapy for castration-resistant prostate cancer. $\mathrm{N}$ Engl J Med. 2010; 363:411-422.

4. Drake CG. Prostate cancer as a model for tumour immunotherapy. Nat Rev Immunol. 2010; 10:580-593.

5. Higano CS, Corman JM, Smith DC, Centeno AS, Steidle CP, Gittleman M, Simons JW, Sacks N, Aimi J and Small EJ. Phase 1/2 dose-escalation study of a GM-CSF-secreting, allogeneic, cellular immunotherapy for metastatic hormonerefractory prostate cancer. Cancer. 2008; 113:975-984.

6. Madan RA, Mohebtash M, Arlen PM, Vergati M, Rauckhorst M, Steinberg SM, Tsang KY, Poole DJ, Parnes HL, Wright JJ, Dahut WL, Schlom J and Gulley JL. Ipilimumab and a poxviral vaccine targeting prostatespecific antigen in metastatic castration-resistant prostate cancer: a phase 1 dose-escalation trial. Lancet Oncol. 2012; 
13:501-508.

7. Slovin SF, Higano CS, Hamid O, Tejwani S, Harzstark A, Alumkal JJ, Scher HI, Chin K, Gagnier P, McHenry MB and Beer TM. Ipilimumab alone or in combination with radiotherapy in metastatic castration-resistant prostate cancer: results from an open-label, multicenter phase I/II study. Ann Oncol. 2013; 24:1813-1821.

8. Ryan CJ, Elkin EP, Small EJ, Duchane J and Carroll P. Reduced incidence of bony metastasis at initial prostate cancer diagnosis: data from CaPSURE. Urol Oncol. 2006; 24:396-402.

9. Tangen CM, Hussain MH, Higano CS, Eisenberger MA, Small EJ, Wilding G, Donnelly BJ, Schelhammer PF, Crawford ED, Vogelzang NJ, Powell IJ and Thompson IM, Jr. Improved overall survival trends of men with newly diagnosed M1 prostate cancer: a SWOG phase III trial experience (S8494, S8894 and S9346). J Urol. 2012; 188:1164-1169.

10. Glass TR, Tangen CM, Crawford ED and Thompson I. Metastatic carcinoma of the prostate: identifying prognostic groups using recursive partitioning. J Urol. 2003; 169:164169.

11. Vivier E, Tomasello E, Baratin M, Walzer T and Ugolini S. Functions of natural killer cells. Nat Immunol. 2008; 9:503510 .

12. Hanna J, Bechtel P, Zhai Y, Youssef F, McLachlan K and Mandelboim O. Novel insights on human NK cells' immunological modalities revealed by gene expression profiling. J Immunol. 2004; 173:6547-6563.

13. Moretta A, Bottino C, Vitale M, Pende D, Biassoni R, Mingari MC and Moretta L. Receptors for HLA class-I molecules in human natural killer cells. Annu Rev Immunol. 1996; 14:619-648.

14. Moretta A, Sivori S, Vitale M, Pende D, Morelli L, Augugliaro R, Bottino C and Moretta L. Existence of both inhibitory (p58) and activatory (p50) receptors for HLA-C molecules in human natural killer cells. J Exp Med. 1995; 182:875-884.

15. Long EO. Regulation of immune responses through inhibitory receptors. Annu Rev Immunol. 1999; 17:875904.

16. Lopez-Botet M, Perez-Villar JJ, Carretero M, Rodriguez A, Melero I, Bellon T, Llano M and Navarro F. Structure and function of the CD94 C-type lectin receptor complex involved in recognition of HLA class I molecules. Immunol Rev. 1997; 155:165-174.

17. Lanier LL. NK cell recognition. Annu Rev Immunol. 2005; 23:225-274

18. Moretta A, Bottino C, Vitale M, Pende D, Cantoni C, Mingari MC, Biassoni R and Moretta L. Activating receptors and coreceptors involved in human natural killer cell-mediated cytolysis. Annu Rev Immunol. 2001; 19:197223.

19. Bauer S, Groh V, Wu J, Steinle A, Phillips JH, Lanier LL and Spies T. Activation of NK cells and T cells by NKG2D, a receptor for stress-inducible MICA. Science. 1999; 285:727-729.

20. Bryceson YT, March ME, Barber DF, Ljunggren HG and Long EO. Cytolytic granule polarization and degranulation controlled by different receptors in resting NK cells. J Exp Med. 2005; 202:1001-1012.

21. Imai K, Matsuyama S, Miyake S, Suga K and Nakachi K. Natural cytotoxic activity of peripheral-blood lymphocytes and cancer incidence: an 11-year follow-up study of a general population. Lancet. 2000; 356:1795-1799.

22. Fauriat C, Just-Landi S, Mallet F, Arnoulet C, Sainty D, Olive D and Costello RT. Deficient expression of NCR in NK cells from acute myeloid leukemia: Evolution during leukemia treatment and impact of leukemia cells in NCRdull phenotype induction. Blood. 2007; 109:323-330.

23. Costello RT, Knoblauch B, Sanchez C, Mercier D, Le Treut T and Sebahoun G. Expression of natural killer cell activating receptors in patients with chronic lymphocytic leukaemia. Immunology. 2012; 135:151-157.

24. Pietra G, Manzini C, Rivara S, Vitale M, Cantoni C, Petretto A, Balsamo M, Conte R, Benelli R, Minghelli S, Solari N, Gualco M, Queirolo P, Moretta L and Mingari MC. Melanoma cells inhibit natural killer cell function by modulating the expression of activating receptors and cytolytic activity. Cancer Res. 2012; 72:1407-1415.

25. Fregni G, Perier A, Pittari G, Jacobelli S, Sastre X, Gervois N, Allard M, Bercovici N, Avril MF and Caignard A. Unique functional status of natural killer cells in metastatic stage IV melanoma patients and its modulation by chemotherapy. Clin Cancer Res. 2011; 17:2628-2637.

26. Fregni G, Messaoudene M, Fourmentraux-Neves E, Mazouz-Dorval S, Chanal J, Maubec E, Marinho E, Scheer-Senyarich I, Cremer I, Avril MF and Caignard A. Phenotypic and functional characteristics of blood natural killer cells from melanoma patients at different clinical stages. PLoS One. 2013; 8:e76928.

27. Garcia-Iglesias T, Del Toro-Arreola A, Albarran-Somoza B, Del Toro-Arreola S, Sanchez-Hernandez PE, RamirezDuenas MG, Balderas-Pena LM, Bravo-Cuellar A, OrtizLazareno PC and Daneri-Navarro A. Low NKp30, NKp46 and NKG2D expression and reduced cytotoxic activity on NK cells in cervical cancer and precursor lesions. BMC Cancer. 2009; 9:186.

28. Mamessier E, Sylvain A, Thibult ML, Houvenaeghel G, Jacquemier J, Castellano R, Goncalves A, Andre P, Romagne F, Thibault G, Viens P, Birnbaum D, Bertucci F, Moretta A and Olive D. Human breast cancer cells enhance self tolerance by promoting evasion from NK cell antitumor immunity. J Clin Invest. 2011; 121:3609-3622.

29. Mamessier E, Sylvain A, Bertucci F, Castellano R, Finetti P, Houvenaeghel G, Charaffe-Jaufret E, Birnbaum D, Moretta A and Olive D. Human breast tumor cells induce self-tolerance mechanisms to avoid NKG2D-mediated and DNAM-mediated NK cell recognition. Cancer Res. 2011; 
71:6621-6632.

30. Platonova S, Cherfils-Vicini J, Damotte D, Crozet L, Vieillard V, Validire P, Andre P, Dieu-Nosjean MC, Alifano M, Regnard JF, Fridman WH, Sautes-Fridman $\mathrm{C}$ and Cremer I. Profound coordinated alterations of intratumoral NK cell phenotype and function in lung carcinoma. Cancer Res. 2011; 71:5412-5422.

31. Gillard-Bocquet M, Caer C, Cagnard N, Crozet L, Perez M, Fridman WH, Sautes-Fridman C and Cremer I. Lung tumor microenvironment induces specific gene expression signature in intratumoral NK cells. Front Immunol. 2013; $4: 19$.

32. Carrega P, Morandi B, Costa R, Frumento G, Forte G, Altavilla G, Ratto GB, Mingari MC, Moretta L and Ferlazzo G. Natural killer cells infiltrating human nonsmall-cell lung cancer are enriched in CD56 bright CD16(-) cells and display an impaired capability to kill tumor cells. Cancer. 2008; 112:863-875.

33. Schleypen JS, Baur N, Kammerer R, Nelson PJ, Rohrmann K, Grone EF, Hohenfellner M, Haferkamp A, Pohla H, Schendel DJ, Falk CS and Noessner E. Cytotoxic markers and frequency predict functional capacity of natural killer cells infiltrating renal cell carcinoma. Clin Cancer Res. 2006; 12:718-725.

34. Rusakiewicz S, Semeraro M, Sarabi M, Desbois M, Locher C, Mendez R, Vimond N, Concha A, Garrido F, Isambert N, Chaigneau L, Le Brun-Ly V, Dubreuil P, Cremer I, Caignard A, Poirier-Colame V, et al. Immune infiltrates are prognostic factors in localized gastrointestinal stromal tumors. Cancer Res. 2013; 73:3499-3510.

35. Delahaye NF, Rusakiewicz S, Martins I, Menard C, Roux S, Lyonnet L, Paul P, Sarabi M, Chaput N, Semeraro M, Minard-Colin V, Poirier-Colame V, Chaba K, Flament C, Baud V, Authier H, et al. Alternatively spliced NKp30 isoforms affect the prognosis of gastrointestinal stromal tumors. Nat Med. 2011; 17:700-707.

36. Arnon TI, Markel G, Bar-Ilan A, Hanna J, Fima E, Benchetrit F, Galili R, Cerwenka A, Benharroch D, SionVardy N, Porgador A and Mandelboim O. Harnessing soluble NK cell killer receptors for the generation of novel cancer immune therapy. PLoS One. 2008; 3:e2150.

37. Gannon PO, Poisson AO, Delvoye N, Lapointe R, MesMasson AM and Saad F. Characterization of the intraprostatic immune cell infiltration in androgen-deprived prostate cancer patients. J Immunol Methods. 2009; 348:917.

38. Brandt CS, Baratin M, Yi EC, Kennedy J, Gao Z, Fox B, Haldeman B, Ostrander CD, Kaifu T, Chabannon C, Moretta A, West R, Xu W, Vivier E and Levin SD. The B7 family member B7-H6 is a tumor cell ligand for the activating natural killer cell receptor NKp30 in humans. $\mathrm{J}$ Exp Med. 2009; 206:1495-1503.

39. Fiegler N, Textor S, Arnold A, Rolle A, Oehme I, Breuhahn K, Moldenhauer G, Witzens-Harig M and Cerwenka A. Downregulation of the activating NKp30 ligand B7-H6 by HDAC inhibitors impairs tumor cell recognition by NK cells. Blood. 2013; 122:684-693.

40. Sivori S, Falco M, Marcenaro E, Parolini S, Biassoni R, Bottino C, Moretta L and Moretta A. Early expression of triggering receptors and regulatory role of 2B4 in human natural killer cell precursors undergoing in vitro differentiation. Proc Natl Acad Sci U S A. 2002; 99:45264531.

41. Hou S, Zheng X, Wei H, Tian Z and Sun R. Recombinant soluble CD226 protein directly inhibits cancer cell proliferation in vitro. Int Immunopharmacol. 2014; 19:119126.

42. Moretta L, Bottino C, Pende D, Castriconi R, Mingari MC and Moretta A. Surface NK receptors and their ligands on tumor cells. Semin Immunol. 2006; 18:151-158.

43. Lundholm M, Schroder M, Nagaeva O, Baranov V, Widmark A, Mincheva-Nilsson L and Wikstrom P. Prostate tumor-derived exosomes down-regulate NKG2D expression on natural killer cells and CD8+ T cells: mechanism of immune evasion. PLoS One. 2014; 9:e108925.

44. Elboim M, Gazit R, Gur C, Ghadially H, Betser-Cohen G and Mandelboim O. Tumor immunoediting by NKp46. J Immunol. 2010; 184:5637-5644.

45. Carlsten M, Norell H, Bryceson YT, Poschke I, Schedvins K, Ljunggren HG, Kiessling R and Malmberg KJ. Primary human tumor cells expressing CD155 impair tumor targeting by down-regulating DNAM-1 on NK cells. J Immunol. 2009; 183:4921-4930.

46. Castriconi R, Cantoni C, Della Chiesa M, Vitale M, Marcenaro E, Conte R, Biassoni R, Bottino C, Moretta L and Moretta A. Transforming growth factor beta 1 inhibits expression of NKp30 and NKG2D receptors: consequences for the NK-mediated killing of dendritic cells. Proc Natl Acad Sci U S A. 2003; 100:4120-4125.

47. Lee JC, Lee KM, Kim DW and Heo DS. Elevated TGFbeta1 secretion and down-modulation of NKG2D underlies impaired NK cytotoxicity in cancer patients. J Immunol. 2004; 172:7335-7340.

48. Sharma J, Gray KP, Harshman LC, Evan C, Nakabayashi M, Fichorova R, Rider J, Mucci L, Kantoff PW and Sweeney CJ. Elevated IL-8, TNF-alpha, and MCP-1 in men with metastatic prostate cancer starting androgendeprivation therapy (ADT) are associated with shorter time to castration-resistance and overall survival. Prostate. 2014; 74:820-828.

49. Koo KC, Shim DH, Yang CM, Lee SB, Kim SM, Shin TY, Kim KH, Yoon HG, Rha KH, Lee JM and Hong SJ. Reduction of the CD16(-)CD56bright NK cell subset precedes NK cell dysfunction in prostate cancer. PLoS One. 2013; 8:e78049.

50. Glasner A, Ghadially H, Gur C, Stanietsky N, Tsukerman $\mathrm{P}$, Enk $\mathrm{J}$ and Mandelboim O. Recognition and prevention of tumor metastasis by the NK receptor NKp46/NCR1. J Immunol. 2012; 188:2509-2515. 
51. Halfteck GG, Elboim M, Gur C, Achdout H, Ghadially H and Mandelboim O. Enhanced in vivo growth of lymphoma tumors in the absence of the NK-activating receptor NKp46/ NCR1. J Immunol. 2009; 182:2221-2230.

52. Gandaglia G, Karakiewicz PI, Briganti A, Passoni NM, Schiffmann J, Trudeau V, Graefen M, Montorsi F and Sun M. Impact of the Site of Metastases on Survival in Patients with Metastatic Prostate Cancer. Eur Urol. 2014.

53. Ost P, Decaestecker K, Lambert B, Fonteyne V, Delrue L, Lumen N, Ameye F and De Meerleer G. Prognostic factors influencing prostate cancer-specific survival in non-castrate patients with metastatic prostate cancer. Prostate. 2014; 74:297-305.

54. McKay K, Moore PC, Smoller BR and Hiatt KM. Association between natural killer cells and regression in melanocytic lesions. Hum Pathol. 2011; 42:1960-1964.

55. Mamessier E, Pradel LC, Thibult ML, Drevet C, Zouine A, Jacquemier J, Houvenaeghel G, Bertucci F, Birnbaum D and Olive D. Peripheral blood NK cells from breast cancer patients are tumor-induced composite subsets. J Immunol. 2013; 190:2424-2436. 